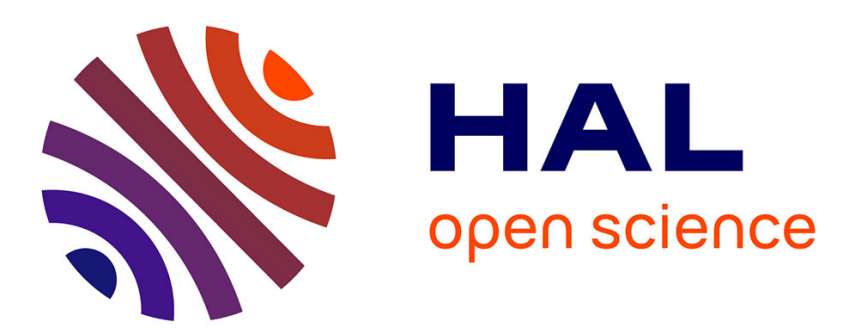

\title{
Use of grating theories in integrated optics
}

Eric Silberstein, Philippe Lalanne, Jean-Paul Hugonin, Qing Cao

\section{To cite this version:}

Eric Silberstein, Philippe Lalanne, Jean-Paul Hugonin, Qing Cao. Use of grating theories in integrated optics. Journal of the Optical Society of America. A Optics, Image Science, and Vision, 2001, 18 (11), pp.2865-2875. hal-00867923

\section{HAL Id: hal-00867923 \\ https://hal-iogs.archives-ouvertes.fr/hal-00867923}

Submitted on 30 Sep 2013

HAL is a multi-disciplinary open access archive for the deposit and dissemination of scientific research documents, whether they are published or not. The documents may come from teaching and research institutions in France or abroad, or from public or private research centers.
L'archive ouverte pluridisciplinaire HAL, est destinée au dépôt et à la diffusion de documents scientifiques de niveau recherche, publiés ou non, émanant des établissements d'enseignement et de recherche français ou étrangers, des laboratoires publics ou privés. 


\title{
Use of grating theories in integrated optics
}

\author{
Eric Silberstein, Philippe Lalanne, Jean-Paul Hugonin, and Qing Cao \\ Laboratoire Charles Fabry de l'Institut d'Optique, Centre National de la Recherche Scientifique, B.P. 147, \\ F-91403 Orsay Cedex, France
}

Received January 25, 2001; revised manuscript received May 8, 2001; accepted May 9, 2001

\begin{abstract}
Recently [Opt. Lett. 25, 1092 (2000)], two of the present authors proposed extending the domain of applicability of grating theories to aperiodic structures, especially the diffraction structures that are encountered in integrated optics. This extension was achieved by introduction of virtual periodicity and incorporation of artificial absorbers at the boundaries of the elementary cells of periodic structures. Refinements and extensions of that previous research are presented. Included is a thorough discussion of the effect of the absorber quality on the accuracy of the computational results, with highly accurate computational results being achieved with perfectly matched layer absorbers. The extensions are concerned with the diversity of diffraction waveguide problems to which the method is applied. These problems include two-dimensional classical problems such as those involving Bragg mirrors and grating couplers that may be difficult to model because of the length of the components and three-dimensional problems such as those involving integrated diffraction gratings, photonic crystal waveguides, and waveguide airbridge microcavities. Rigorous coupled-wave analysis (also called the Fourier modal method) is used to support the analysis, but we believe that the approach is applicable to other grating theories. The method is tested both against available numerical data obtained with finite-difference techniques and against experimental data. Excellent agreement is obtained. A comparison in terms of convergence speed with the finite-difference modal method that is widely used in waveguide theory confirms the relevancy of the approach. Consequently, a simple, efficient, and stable method that may also be applied to waveguide and grating diffraction problems is proposed. (C) 2001 Optical Society of America
\end{abstract}

OCIS codes: 050.1950, 050.1970, 130.01.30, 050.1960, 050.1940.

\section{INTRODUCTION}

Numerical characterization and modeling of guided-wave passive components have been important research topics in the past four decades. This is so because of increased research and development in millimeter-wave and photonic integrated circuits. In the early 1960 s, efforts were devoted to establishing the foundations of waveguide theory. In the late $1980 \mathrm{~s}$, more than a dozen numerical methods of analyzing these components were invented and the somewhat more classic methods were refined. ${ }^{1}$ Most of the existing methods have been known from that time. The development of waveguide theory today continues to respond to the demands of the increasingly widespread applications of integrated optics. The theory features improvements of the existing methods in terms of numerical stability, convergence speed, and versatility. Most of this can be said for grating theories as well. ${ }^{2}$ Clearly, because the theory of electromagnetism and waveguide and grating methods rely on the same foundation, they share many common features. However, probably because they are directed to the modeling of different structures and because the applications are rather different, it appears that these theories were developed mainly independently and that clear bridges between them were rarely made in the literature. We do so here and propose a simple, efficient, and stable theory that may apply to grating and to waveguide diffraction problems, as well.

To illustrate our purpose, we consider the rigorous coupled wave analysis ${ }^{3}$ (RCWA) hereafter. RCWA is a frequency-domain method of waveguide analysis that is widely used in grating theory. It was developed over many years; see Refs. 2 and 3 and the reference therein and in the related research on the so-called differential method. ${ }^{4}$ RCWA is based on the computations of the grating modes as eigenvectors and on Fourier expansions for the permittivity and for the electromagnetic fields inside the grating region. It is also called the Fourier modal method by many authors. Perhaps the main difference between waveguide and grating theories concerns the outgoing and ingoing wave conditions inside the inhomogeneous region. In grating theory, because of periodicity, the electromagnetic fields are pseudoperiodic functions and the outgoing and ingoing wave conditions are strictly fulfilled. In waveguide theory, a computational domain delimited by absorbing boundaries is defined. How well the outgoing and ingoing wave conditions can be satisfied depends on the quality of the absorbers; see Ref. 5 in the context of finite-difference techniques. As was shown by some of the present authors, ${ }^{6}$ the extension of the RCWA to modeling waveguide-diffraction problems relies on the introduction of absorbers. It is worth mentioning that the kinds of absorber (metallic, anisotropic, magnetic...) that can be efficiently incorporated into a given method depend on the method itself. Hereafter, we restrict ourselves to absorbers that can be numerically implemented efficiently with minor modification of the RCWA.

In Section 2 we describe the main idea of our approach to coupled-wave analysis. It is assumed that the reader is familiar with RCWA, ${ }^{3}$ and with its enhanced version ${ }^{7}$ for TM polarization and its extension to crossed gratings. ${ }^{8}$ Section 2 also includes a brief description of the $S$-matrix propagation algorithm ${ }^{9}$ used for analyzing multilayer structures and an extension of the RCWA to anisotropic and magnetic media. This extension is motivated not by 
the analysis of waveguides with magnetic or anisotropic material but by the performance enhancement that is achieved with magnetic and anisotropic absorbers. The influence of the absorbers on the computational accuracy of the method is studied in Section 3. Sections 4 and 5 provide numerical results for two- and three-dimensional structures. Section 4 is concerned with classic problems, namely, those concerning distributed Bragg reflectors and grating couplers. In Section 5 we consider more up-todate subjects such as the diffraction of guided waves by integrated gratings, photonic-crystal waveguides, and waveguide air-bridge microcavities. The method is tested both against available numerical data and against experimental data for photonic-crystal waveguides. In all cases, excellent agreement is achieved. In Section 6 we conclude the paper and discuss some possible extensions of this research.

\section{SOLVING WAVEGUIDE-DIFFRACTION PROBLEMS WITH RIGOROUS COUPLED-WAVE ANALYSIS}

Figure 1 shows one possible waveguide geometry, which we use here to illustrate the method of solving twodimensional waveguide diffraction problems. It represents a planar waveguide structure with a binary corrugation profile. In every homogeneous section of the waveguide geometry the permittivity and the permeability depend only on the $x$ (vertical) coordinate. The structure is invariant in the $y$ direction, which is perpendicular to the figure. Both metallic and dielectric materials may be considered. The corrugated waveguide is assumed to be illuminated from the input region by a guided wave, the fundamental $\mathrm{TE}_{0}$ or $\mathrm{TM}_{0}$ mode, for instance. $\mathrm{TE}$ and TM polarizations refer to situations for which the electric and magnetic fields, respectively, are parallel to the $y$ direction. These two states of polarization can be handled independently. The wavelength in vacuum of the incident mode is denoted $\lambda\left(k_{0}=2 \pi / \lambda\right)$. Our goal is to compute the light that is scattered in the substrate and the superstrate, reflected in the input region, or transmitted in the output region.

A. Artificial Periodicity, Absorbers, and Fourier Series To analyze such waveguide geometries with RCWA we introduce $^{6}$ an artificial periodicity along the $x$ coordinate, thus virtually replacing the actual waveguide isolated in space with a periodic waveguide structure; see Fig. 2. This structure defines a period (or a computational box) $w$

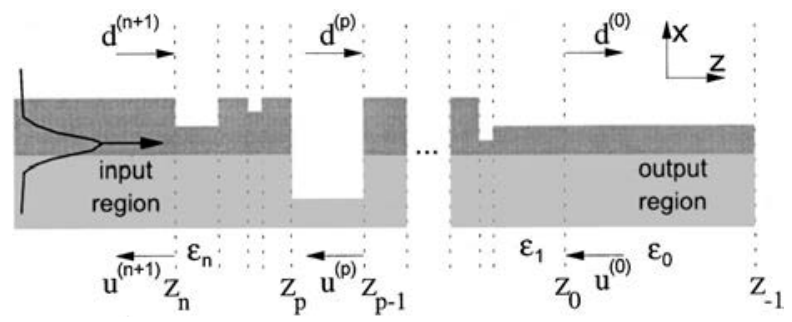

Fig. 1. General layered waveguide diffraction. Electromagnetic fields are computed as eigenmodes in every layer, and the field in each layer is represented as a superposition of left- and right-propagating modes with amplitudes $\mathbf{u}^{(p)}$ and $\mathbf{d}^{(p)}$.

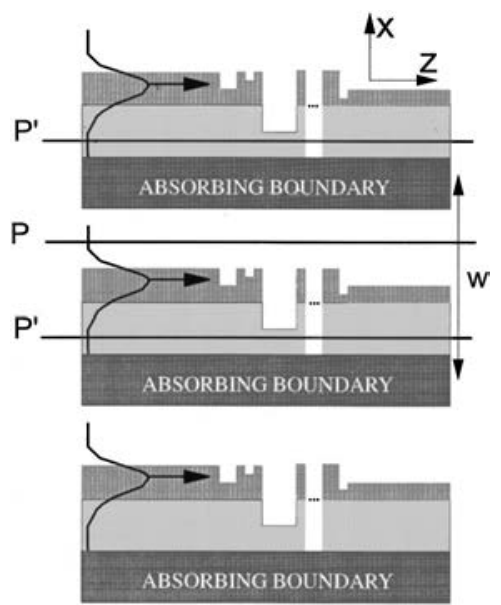

Fig. 2. Artificial periodicity of the waveguide and adjunction of absorbers between waveguide structures.

in the $x$ direction. With the introduction of this periodicity (which we call "periodization"), RCWA can be applied to solve the diffraction problem. For the electromagnetic solution of the periodic waveguide geometry and that of the isolated waveguide structures to be identical, absorbers have to be incorporated between the waveguides. These absorbers, represented by dark rectangles in Fig. 2, are used to satisfy the ingoing wave condition at planes $\mathrm{P}$ and $\mathrm{P}^{\prime}$, two planes that surround the waveguides. At plane $\mathrm{P}$ or $\mathrm{P}^{\prime}$ the ingoing wave condition stipulates that no waves propagate down or up, respectively. It is easily verified that satisfying the ingoing wave condition amounts to considering that the absorber does not transmit the light scattered by the corrugation, thus making the neighboring waveguides of the periodic waveguide structure electromagnetically independent of each other, and does not reflect the scattered light, thus preventing electromagnetic contamination inside a single period.

As is shown in Section 3 below, the absorbers are composed of two independent parts, one placed in contact with the superstrate and the second in contact with the substrate. Note that the absorbers' permittivity and permeability depend on the refractive indices of the superstrate and the substrate in only an analytical way. Thus the absorbers are designed straightforwardly; see Section 3 below. Each absorber part absorbs light. This absorption guarantees that the electromagnetic field quantities are null at the border of the computational box. Thus we use the Fourier series to expand periodic electromagnetic field quantities that are null at the borders of the computational box and that are identical to the real solution of the isolated waveguide-diffraction problem outside the absorber regions.

With the inclusion of the absorbing layers that results in modification of the index distribution profile, the waveguide modes and their effective indices in every uniform section are computed as eigenvectors and eigenvalues. Then the $S$-matrix algorithm is used to link the field amplitudes without any numerical instability, as we discuss in Subsections 2.B and 2.C.

Generally speaking, the proposed method belongs to the class of modal spectral methods. ${ }^{1}$ The originality of our approach lies in the use of Fourier series to expand 
the modes in every uniform section of the waveguide. Let us first note that, because the same expansion bases are used in every section, mode-matching techniques ${ }^{1}$ are straightforwardly applied to matching the fields on both sides of two discontinuities of adjacent sections. Second, it is worth mentioning that, since the recent discovery made by the authors of Refs. 7 and 10, the use of Fourier series in electromagnetic computations is placed on a firm mathematical foundation ${ }^{11}$ and permits high-convergence performance for lamellar gratings, ${ }^{7,8,10}$ for continuousprofile gratings, ${ }^{12}$ and for photonic-crystal-related problems. ${ }^{13}$ Thus we believe that Fourier series represent a simple and relevant expansion basis not only for grating theories when they appear naturally but also for more-general aperiodic cases. For instance, as was shown in Subsections 3.B and 5 of Ref. 14, modal theories that rely on Fourier series outperform finite-difference techniques in terms of convergence performance, even when permittivity interpolation schemes and nonuniform sampling are implemented in the finite-difference approach. In Section 5 of this paper similar results are reported.

\section{B. Eigenproblem Formulation with Magnetic and Anisotropic Media}

As we have just said, the approach to eigenproblem formulation is based on the adjunction of absorbing boundaries on a periodic waveguide structure. The absorbing boundaries are purely numerical layers and consequently may have an arbitrary permittivity or permeability distribution. Previously, ${ }^{6}$ Lalanne and Silberstein considered simple gradient-index absorbers. Here we consider more-sophisticated absorbers, which are composed of magnetic and anisotropic media. Using these types of absorber guarantees that, when a propagating or evanescent plane wave is incident from the superstrate or substrate onto the absorber, no light is reflected. In fact, these absorbers are a specific type of perfectly matched layers $^{15}$ (PMLs) that can be implemented efficiently with RCWA. Adding magnetic and anisotropic properties provides the opportunity for access to a supplementary degree of freedom and for improving the quality of the absorbers. The TM polarization case is described in detail hereafter. TE polarization is deduced by use of the symmetry of Maxwell's equations, as is briefly outlined at the end of this section.

All uniform sections of the waveguide geometry, including the input and output regions, are treated similarly. Hereafter the superscript $(p), p=0,1, \ldots, n+1$, refers to the uniform section under consideration. We assume that the materials in section $p$ are magnetic with relative permeability $\mu(x)$ and anisotropic with a relative tensor of permittivity defined by $\varepsilon_{x x}(x)$ and $\varepsilon_{z z}(x)$, all the other tensor coefficients being null. From the curl Maxwell equations, after eliminating the electric-field components $E_{x}^{(p)}$ and $E_{z}^{(p)}$, we obtain

$$
\frac{\partial^{2} H_{y}^{(p)}}{\partial z^{2}}=-k_{0}^{2} \varepsilon_{x x}(x) \mu(x) H_{y}^{(p)}-\varepsilon_{x x}(x) \frac{\partial}{\partial x}\left[\frac{1}{\varepsilon_{z z}(x)} \frac{\partial H_{y}^{(p)}}{\partial x}\right],
$$

where $H_{y}^{(p)}$ is the $y$ component of the magnetic field in section $(p)$. As for classic RCWA, the electric and magnetic fields are expanded in Fourier series. For the $y$ component of the magnetic field we have

$$
H_{y}^{(p)}=\sum_{m=-\infty}^{\infty} U_{m}(z) \exp (j m K x),
$$

where $K=2 \pi / w, j^{2}=-1$, and $U_{m}$ is the unknown normalized amplitude of the $m$ th space-harmonic field. Note that the electromagnetic field is looked for as a purely periodic function of the $x$ coordinate and not as a pseudoperiodic one as in classic RCWA. We achieve this simply by setting the angle of incidence equal to zero in standard RCWA code. To perform the computation we must truncate the Fourier series in Eq. (2). We denote by $[\mathbf{U}]$ the vector formed by the finite set of the normalized amplitudes retained for the computation. Let us denote by $\mathbf{E}, \mathbf{A}$, and $\mathbf{B}$ the Toeplitz matrices associated with Fourier coefficients of $\varepsilon_{z z}(x), 1 / \varepsilon_{x x}(x)$, and $\mu(x)$, respectively. By substituting Eq. (2) into Eq. (1) and identifying terms in the Fourier basis, we find that the set of second-order differential equations for the magnetic field can be put in the compact form

$$
\left(\frac{\partial^{2} U}{\partial z^{2}}\right)=k_{0}^{2}\left[\mathbf{A}^{-1}\left(\mathbf{K}_{x} \mathbf{E}^{-1} \mathbf{K}_{x}-\mathbf{B}\right)\right][\mathbf{U}]
$$

where $\mathbf{K}_{x}$ is a diagonal matrix and the $(m, m)$ element is $m K / k_{0}$. It is easy to determine that the matrices involved in Eq. (3) verify the Fourier factorization rules of Ref. 11 and that Eq. (3) reduces to Eq. (9) of Ref. 7 for nonmagnetic and isotropic media ( $\mathbf{B}$ is the identity matrix in this case). Although the matrix involved in Eq. (3) is more complex than that used for isotropic and nonmagnetic media [see Eq. (9) of Ref. 7 for comparison], this additional complexity remains weak (only two additional Toeplitz matrices have to be computed) and is worth being implemented, as it allows one to obtain accurate results for small $w$ values; see Section 3 below for more details. From Eq. (3), the modes $W_{m}^{(p)}$ and the propagation constants $\lambda_{m}^{(p)}$ in every section $p$ are computed as the eigenvectors and the square roots of the eigenvalues of the matrix $\mathbf{A}^{-1}\left(\mathbf{K}_{x} \mathbf{E}^{-1} \mathbf{K}_{x}-\mathbf{B}\right)$. Thus, in each section, electromagnetic field $H_{y}^{(p)}$ is looked for as a superposition of modes

$$
\begin{aligned}
U_{p}(z)= & \sum_{m} W_{m}^{(p)}\left\{u_{m}^{(p)}\left\lfloor-k_{0} \lambda_{m}^{(p)}\left(z-z_{p-1}\right)\right\rfloor\right. \\
& \left.+d_{m}^{(p)} \exp \left\lfloor k_{0} \lambda_{m}^{(p)}\left(z-z_{p-1}\right)\right\rfloor\right\},
\end{aligned}
$$

where $\mathbf{u}^{(p)}$ and $\mathbf{d}^{(p)}$ are column vectors whose elements are the amplitudes of the modes propagating backward (in the $-z$ direction) and forward (in the $z$ direction), respectively. The unknown normalized amplitudes $S_{p}$ of the $p$ th $x$ component of the electric field, defined by

$$
E_{x}^{(p)}=j\left(\frac{\mu_{0}}{\varepsilon_{0}}\right)^{1 / 2} \sum_{m=-\infty}^{+\infty} S_{x m} \exp (j m K x)
$$

and used to match the boundary conditions at planes $z$ $=z_{p}$ and $z=z_{p+1}$, are simply 


$$
\begin{aligned}
S_{x p}(z)= & \sum_{m} V_{m}^{(p)}\left\{u_{m}^{(p)} \exp \left\lfloor-k_{0} \lambda_{m}^{(p)}\left(z-z_{p-1}\right)\right\rfloor\right. \\
& \left.-d_{m}^{(p)} \exp \left\lfloor k_{0} \lambda_{m}^{(p)}\left(z-z_{p-1}\right)\right\rfloor\right\},
\end{aligned}
$$

where $\mathbf{v}_{m}^{(p)}$ is the $m$ th column vector of matrix $\mathbf{V}^{(p)}$ $=\mathbf{A} \mathbf{W}^{(p)} \lambda^{(p)}, \lambda^{(p)}$ is a diagonal matrix, and the $(m, m)$ element is $\lambda_{m}^{(p)}$.

The TE polarization case is easily deduced from the previous analysis by use of the symmetry of Maxwell's equations, $\varepsilon \leftrightarrow \mu$ and $H \leftrightarrow E$. For instance, the eigenmatrix becomes $\mathbf{C}^{-1}\left(\mathbf{K}_{x} \mathbf{D}^{-1} \mathbf{K}_{x}-\mathbf{F}\right)$, where $\mathbf{C}, \mathbf{D}$, and $\mathbf{F}$ are the Toeplitz matrices of $1 / \mu_{x x}, \mu_{z z}$, and $\varepsilon(x)$, respectively. The corresponding $\mathbf{V}$ vector that is proportional to the $x$ component of the magnetic field is $\mathbf{C W} \mathbf{W}^{(p)} \lambda^{(p)}$. It is noteworthy that the symmetry of Maxwell's equations is reflected in the numerical code implementations. Once the code is implemented for a given polarization, we can obtain the other polarization case straightforwardly just by interchanging the $\varepsilon(x)$ and $\mu(x)$ distributions.

\section{S-Matrix Algorithm}

Once the eigenmodes and their propagating constants are known in every layer, we use an $S$-matrix algorithm to compute the $\mathbf{u}^{(p)}$ and $\mathbf{d}^{(p)}$ amplitude vectors. The difference between the research reported here and the $S$-matrix implementation used for classic grating theories is rather small: In grating theories, a Rayleigh expansion is used to describe the electromagnetic field in the input and output homogeneous regions. In the present case, the field expansions in the input and output regions are periodic Floquet expansions, as for all the layers of the corrugated region. This difference even simplifies the implementation of the $S$-matrix algorithm.

The $S$-matrix algorithm is a stable and efficient algorithm that does not exhibit any numerical instabilities with the growing exponential terms in Eqs. (4) and (6). To avoid repetition of already published results, we exactly follow the notation of Ref. 9, where many details of the stability and efficiency of propagation algorithms are discussed. We define the $S$-matrix $\mathbf{S}^{(p)}$ that links the waves in the section $p+1$ and those of medium 0 in this way:

$$
\left[\begin{array}{c}
u^{(p+1)} \\
d^{(0)}
\end{array}\right]=S^{(p)}\left[\begin{array}{c}
u^{(0)} \\
d^{(p+1)}
\end{array}\right]
$$

with

$$
S^{(p)}=\left[\begin{array}{cc}
T_{u u}^{(p)} & R_{u d}^{(p)} \\
R_{d u}^{(p)} & T_{d d}^{(p)}
\end{array}\right] .
$$

The transmission and reflection submatrices in Eq. (8) are computed in a recursive way. If no light is impinging on the corrugated waveguide from the output region $\left[\mathbf{u}^{(0)}=\mathbf{0}\right.$ ], only $\mathbf{R}_{u d}^{(p)}$ and $\mathbf{T}_{d d}^{(p)}$ are required for computing $\mathbf{u}^{(p+1)}$ and $\mathbf{d}^{(0)}$. These recursion formulas are ${ }^{9}$

$$
\begin{aligned}
\mathbf{R}_{u d}^{(p)} & =r_{u d}^{(p)}+t_{u u}^{(p)} R_{u d}^{(p-1)}\left[1-r_{d u}^{(p)} R_{u d}^{(p-1)}\right]^{-1} t_{d d}^{(p)}, \\
\mathbf{T}_{d d}^{(p)} & =T_{d d}^{(p-1)}\left[1-r_{d u}^{(p)} R_{u d}^{(p-1)}\right]^{-1} t_{d d}^{(p)} .
\end{aligned}
$$

In Eqs. (9) the matrices denoted by lowercase letters are the reflection and transmission submatrices of the $S$ ma- trix that relate the waves in the two adjacent layers $p$ and $p+1$. They are given by

$$
\begin{aligned}
\mathbf{t}_{u u}^{(p)}= & 2\left[\mathbf{W}^{(p)-1} \mathbf{W}^{(p+1)}+\mathbf{V}^{(p)-1} \mathbf{V}^{(p+1)}\right]^{-1} \mathbf{X}^{(p)}, \\
\mathbf{r}_{u d}^{(p)}=[ & \mathbf{W}^{(p)-1} \mathbf{W}^{(p+1)} \\
& \left.+\mathbf{V}^{(p)-1} \mathbf{V}^{(p+1)}\right]^{-1}\left[\mathbf{W}^{(p)-1} \mathbf{W}^{(p+1)}\right. \\
& \left.-\mathbf{V}^{(p)-1} \mathbf{V}^{(p+1)}\right] \\
\mathbf{r}_{d u}^{(p)}= & \mathbf{X}^{(p)}\left[\mathbf{V}^{(p+1)-1} \mathbf{V}^{(p)}\right. \\
& \left.+\mathbf{W}^{(p+1)-1} \mathbf{W}^{(p)}\right]^{-1}\left[\mathbf{V}^{(p+1)-1} \mathbf{V}^{(p)}\right. \\
& \left.-\mathbf{W}^{(p+1)-1} \mathbf{W}^{(p)}\right] \mathbf{X}^{(p)}, \\
\mathbf{t}_{d d}^{(p)}= & 2 \mathbf{X}^{(p)}\left[\mathbf{V}^{(p+1)-1} \mathbf{V}^{(p)}+\mathbf{W}^{(p+1)-1} \mathbf{W}^{(p)}\right]^{-1} .
\end{aligned}
$$

where $\mathbf{X}^{(p)}$ is a diagonal matrix and the $(m, m)$ coefficient is $\exp \left\lfloor-\lambda_{m}^{(p)}\left(z_{p-1}-z_{p}\right)\right\rfloor$. We initialize the matrix $S$-recursion by setting $S^{(-1)}$ equal to the identity matrix. Matrices $\mathbf{S}^{(p)}$ are computed recursively until matrix $\mathbf{S}^{(n)}$ is obtained. The amplitudes of the modes propagating backward in the input region, $\mathbf{u}^{(n+1)}$, and forward in the output region, $\mathbf{d}^{(0)}$, are obtained from Eq. (7), where $\mathbf{u}^{(0)}$ $=\mathbf{0}$ and $\mathbf{d}^{(n+1)}$ represent the components of the incident wave expanded on the complete set of modes supported by the waveguide of the input region. Under the usual assumption that the corrugated waveguide is illuminated by the fundamental $\mathrm{TE}_{0}$ or $\mathrm{TM}_{0}$ mode with unitary amplitude, all components of vector $\mathbf{d}^{(n+1)}$ are zero, except the $i$ th component $d_{i}^{(n+1)}$, which is equal to 1 , where $i$ is the eigenmatrix column number that corresponds to the fundamental mode. If we define the reflected intensity $R_{m}$ by the amount of light that is backreflected into mode $m$, $R_{m}$ is simply equal to $\left|u_{m}^{(n+1)}\right|^{2}$ times the ratio between the time-averaged Poynting-vector $z$ components of the reflected mode $m$ and that of the fundamental input mode:

$$
R_{m}=\left|u_{m}^{(n+1)}\right|^{2}\left\langle W_{m}^{(n+1)}, V_{m}^{(n+1)}\right\rangle /\left\langle W_{i}^{(n+1)}, V_{i}^{(n+1)}\right\rangle,
$$

where $\langle x, y\rangle$ is the absolute value of the imaginary part of the scalar product of $x$ and the complex conjugate of $y$. Similarly, for the transmitted intensity $T_{m}$ into mode $m$ we have

$$
T_{m}=\left|d_{m}^{(0)}\right|^{2}\left\langle W_{m}^{(0)}, V_{m}^{(0)}\right\rangle /\left\langle W_{i}^{(n+1)}, V_{i}^{(n+1)}\right\rangle .
$$

The programmer's effort to transform a grating RCWA code equipped with the $S$-matrix algorithm into a waveguide code is rather minimal. The main effort, which consists in modifying the permittivity and permeability distributions to include the absorbers, is the more intensive, but the modifications are straightforward.

\section{INFLUENCE OF THE ABSORBERS ON NUMERICAL ACCURACY}

Perfectly absorbing boundaries that exactly satisfy the ingoing wave condition provide the actual physical solution to the isolated waveguide diffraction problem. Specification of optimal absorbers with small thickness is a challenge for the computational electromagnetic community, 
and much effort is being devoted to determining it, especially for finite-difference techniques. ${ }^{16}$ However, absorbing boundaries are not perfect in practical situations and cannot be made arbitrarily thin. Consequently, the effect on the accuracy and on the convergence performance of the absorbers operating with small computational window sizes ( $w$ in the present study) has to be studied. It is worth noting that this effect depends on the numerical tool used and that increasing $w$ is detrimental to convergence. Hereafter we consider absorbers that are easily implemented with the Fourier modal approach described in Subsection 2.A.

To study the various absorbers we consider the waveguide-diffraction problem depicted in Fig. 3. The corrugated waveguide is composed of a $300-\mathrm{nm}$-wide core (refractive index, 3.5) with claddings with refractive indices of 2.9 for the substrate and 1 for the cover. Two identical 150-nm-wide slits are etched into this waveguide. They are separated by a 150 -nm-wide spacer, and the slits are $300 \mathrm{~nm}$ deep. The waveguide is illuminated by its $\mathrm{TE}_{0}$ or $\mathrm{TM}_{0}$ fundamental mode, with a $975-\mathrm{nm}$ wavelength. Inasmuch as the size and the depth of the slits guarantee that a large amount of light is scattered toward the absorbers, this structure is useful for the study of the effect of the absorbers on accuracy of the algorithm.

We tested several absorbers. We report on only three of them. These absorbers are described for TM polarization; the TE polarization is straightforwardly deduced. The absorbers are all $\lambda / 2$ thick, and each is composed of two parts with identical $\lambda / 4$ thickness. Part A1 is placed in contact with the air superstrate. Part A2 is placed in contact with the substrate. The first absorber is isotropic $\left(\varepsilon_{x x}=\varepsilon_{z z}\right)$ and is not magnetic $(\mu=1)$. It has a gradient-index distribution. For parts $\mathrm{A} 1$ and $\mathrm{A} 2$ the refractive indices vary linearly from 1 to $1+j$ and from 2.9 to $2.9+j$, respectively. Similar absorbers were considered in the research reported in Ref. 6. The second absorber is also isotropic $\left(\varepsilon_{x x}=\varepsilon_{z z}=\varepsilon\right)$, with a gradientindex distribution like that of the first absorber, but is magnetic. Part A1 has a refractive index $n=(\varepsilon \mu)^{1 / 2}$ that varies from 1 to $1+j$ with a uniform normalized admittance $(\varepsilon / \mu)^{1 / 2}$ equal to 1 , the normalized admittance of air. This choice guarantees that no light impinging at normal incidence onto absorber A1 from air is reflected. Similarly, part A2 has a refractive index $n=(\varepsilon \mu)^{1 / 2}$ that varies from 2.9 to $2.9+j$, with a uniform normalized admittance $(\varepsilon / \mu)^{1 / 2}$ equal to 2.9 , the normalized admittance of the substrate. For the numerical implementation, the gradient-index absorber profiles are approximated by a staircase profile with 10 slices. The last absorber corresponds to PMLs. It is anisotropic and magnetic. From Ref. 15 it is easily found that, at an interface in the (xyz) plane normal to the $z$ direction between two media [Eqs. (1) and (2)], no reflection occurs for any incidence angle,

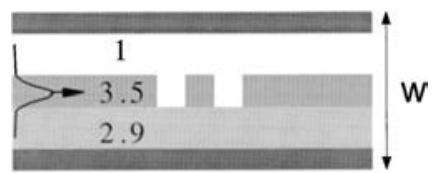

Fig. 3. Investigation of waveguide diffraction for studying the effectiveness of several absorbers.
Table 1. Reflectivity for the WaveguideDiffraction Problem of Fig. 2

\begin{tabular}{|c|c|c|c|c|}
\hline \multirow[b]{2}{*}{$w / \lambda$} & \multirow{2}{*}{$\begin{array}{c}\text { Nonmagnetic } \\
\text { Absorbers } \\
\text { (301 orders) }\end{array}$} & \multirow{2}{*}{$\begin{array}{l}\text { Magnetic } \\
\text { Absorbers } \\
\text { (301 orders) }\end{array}$} & \multicolumn{2}{|c|}{ PML } \\
\hline & & & 301 Orders & 1001 Orders \\
\hline \multicolumn{5}{|c|}{ TE Mode } \\
\hline 1 & 0.3928 & 0.3877 & 0.3952 & 0.39521135 \\
\hline 1.5 & 0.3820 & 0.3825 & 0.3952 & 0.39521131 \\
\hline 2 & 0.3938 & 0.3959 & 0.3952 & 0.39521133 \\
\hline 2.5 & 0.3878 & 0.3859 & 0.3952 & 0.39521135 \\
\hline 3 & 0.3942 & 0.3968 & 0.3952 & 0.39521144 \\
\hline 4 & 0.3937 & 0.3959 & 0.3952 & 0.39521143 \\
\hline 5 & 0.3931 & 0.3944 & 0.3952 & 0.39521172 \\
\hline 6 & 0.3930 & 0.3930 & 0.3952 & 0.39521170 \\
\hline 7 & 0.3934 & 0.3925 & 0.3953 & 0.39521199 \\
\hline \multicolumn{5}{|c|}{ TM Mode } \\
\hline 1 & 0.3584 & 0.3621 & 0.3555 & 0.355480 \\
\hline 1.5 & 0.3582 & 0.3577 & 0.3551 & 0.355482 \\
\hline 2 & 0.3520 & 0.3513 & 0.3555 & 0.355484 \\
\hline 2.5 & 0.3529 & 0.3542 & 0.3556 & 0.355487 \\
\hline 3 & 0.3526 & 0.3520 & 0.3556 & 0.355490 \\
\hline 4 & 0.3533 & 0.3532 & 0.3557 & 0.355499 \\
\hline 5 & 0.3539 & 0.3541 & 0.3557 & 0.355508 \\
\hline 6 & 0.3544 & 03549 & 0.3559 & 0.355521 \\
\hline 7 & 0.3549 & 0.3553 & 0.3560 & 0.355528 \\
\hline
\end{tabular}

provided that $\mu^{(2)} / \mathcal{E}_{z z}^{(2)}=\mu^{(1)} / \mathcal{E}_{z z}^{(1)}$ and $\varepsilon_{x x}^{(2)} \varepsilon_{z z}^{(2)}=\varepsilon_{x x}^{(1)} \varepsilon_{z z}^{(1)}$. Consequently, for part A1 we choose a uniform layer with $\mu=5(1+j), \varepsilon_{z z}=\mu$, and $\varepsilon_{x x}=1 / \mu$. Similarly, part A2 is a uniform layer with $\mu=5(1+j), \varepsilon_{z z}=(2.9)^{2} \mu$, and $\varepsilon_{x x}=(2.9)^{2} / \mu$. The $(1+j)$ terms in the expressions of $\mu$ guarantee that both evanescent and propagating waves are absorbed in the absorber.

Table 1 illustrates the efficiencies of the three absorbers for the two polarizations and for a large range of window sizes. Columns 2-4 were obtained for 301 retained Fourier harmonics. A comparative inspection of these columns clearly shows that the computational accuracy depends mainly on the quality of the absorbers. The best performance is achieved for the PML case of column 4, especially for TE polarization, for which four-digit accuracy in the computed reflectivity is achieved over the entire range of window sizes. An even higher, six-digit accuracy is shown in column 5 for 1001 retained orders. It is evident that the numerical solution is merely independent of size $w$ of the artificial periods. We believe that this independence strongly supports the soundness of the proposed methods, which relies on artificial periodicity for solution of an aperiodic diffraction problem. For TM polarizations the results are less accurate than for TE. However, the accuracy remains high; the relative difference between the reflectivity computed for $w / \lambda=1$ and $w / \lambda$ $=7$ in column 5 of Table 1 is $0.013 \%$. We believe that this lesser degree of accuracy is due only to the fact that, despite the drastic convergence-rate improvement achieved in the research reported in Ref. 7, the RCWA still converges faster for TE than for TM polarization. The method used is of no concern.

It is worth mentioning that one must not conclude from Table 1 that nonmagnetic or magnetic absorbers offer a 
weak two-digit accuracy. Better accuracy can be obtained with thicker absorbers, which reduce the transmission and the reflection. However, an increase in the absorber thickness results in an increase in the artificial period, which is detrimental to the convergence rate. Perhaps the prime benefit of PML absorbers is the high accuracy achieved for small $w / \lambda$ ratios.

\section{NUMERICAL RESULTS FOR TWO- DIMENSIONAL STRUCTURES}

Two-dimensional periodic structures etched into planar waveguides are important components of many optical integrated devices. Examples are Bragg gratings that act as wavelength-selective reflectors, grating-assisted couplers, and passive TE-TM mode converters. In this section the present coupled-wave analysis method is tested for such classic components.

We first consider the planar Bragg-grating waveguide problem of the COST240 project (where COST240 is an acronym of European cooperation in the field of science and technical research). The waveguide is composed of a $2.4-\mu \mathrm{m}$-wide core (refractive index, 1.53) with claddings whose refractive indices are 1 for the cover and 1.52 for the substrate. A TE polarized guided wave $(\lambda$ $=650 \mathrm{~nm})$ is incident onto a weakly modulated Bragg reflector composed of $0.5-\mu \mathrm{m}$-deep grooves etched into the core. For the computation it is assumed that the grating period is $\Lambda=213 \mathrm{~nm}$ and that the groove width is $0.106553 \mu \mathrm{m}$. The main difficulty lies dealing with the large number of periods considered.

Figure 4(a) shows the Bragg-grating reflectivity as a function of the number of periods. The solid curve corresponds to numerical results obtained from the research reported in Ref. 17 by the method of lines. ${ }^{18}$ The stars are data obtained with the present method. Very good agreement between the two approaches is achieved; the maximum relative discrepancy for the reflectivity is less than $1 \%$. The CPU times (in seconds) required for computation with the present method are indicated within brackets in the Figure. We obtained these short CPU
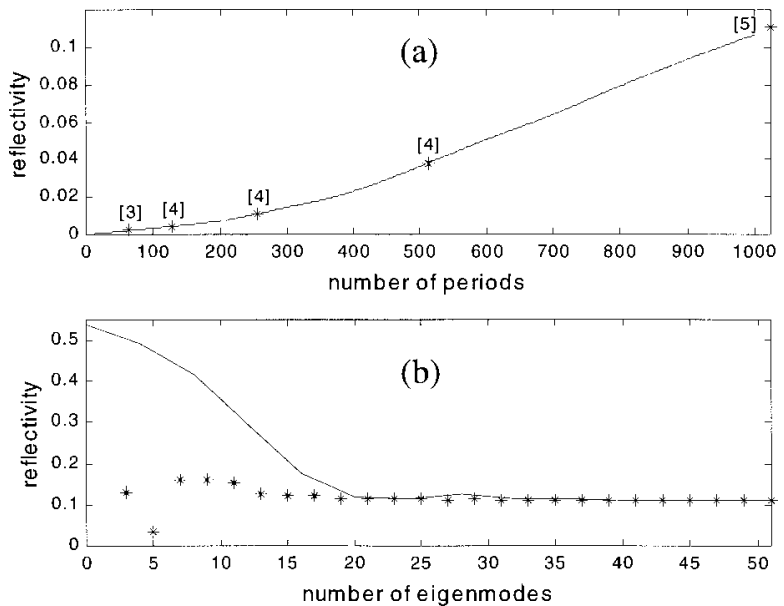

Fig. 4. Comparison of the method of lines (solid curves) and the present method (stars) for a Bragg reflector. (a) Reflectivity computed as a function of the number of periods. (b) Reflectivity for 1024 periods computed as a function of the number of eigenmodes retained for the computation. times by exploiting the periodicity of the Bragg grating in the $z$ direction. More explicitly, if we denote by $S_{p}$ the $S$-matrix associated with one period of the structure, the $S$-matrix $S^{(N)}$ associated with $N$ periods is simply the product $S_{p} * S_{p} \ldots * S_{p}, N$ times, where the star product is defined as in Ref. 9. For increasing the computational speed, we simply expand the product $S_{p} * S_{p} \ldots * S_{p}$ in a series of powers of 2 :

$$
S^{(N)}=\sum_{p=1,2 \ldots} a_{p} S_{p}^{\left(2^{p}\right)},
$$

where $a_{p}$ is 0 or 1 . With this approach, the computation of reflection and transmission for 1024 periods, for instance, requires only the computation of $S^{(2)}=S_{p} * S_{p}$, $S^{(4)}=S^{(2) *} S^{(2)}, \ldots, S^{(512)}=S^{(256) *} S^{(256)}$, and $S^{(1024)}$ $=S^{(512)} * S^{(512)}$. Thus only ten $S$-matrix products are involved. The CPU time required for computation of the ten products is not significant compared with the CPU time required for solution of the two eigenmode problems in each uniform section. Thus the total CPU time is only weakly dependent on the number of periods of the Bragg reflector.

The stars in Fig. 4(b) show the reflectivity of the 1024$\Lambda$-period reflector computed with the present method as a function of the number of eigenmodes retained for the computation. Results were obtained for a 9.3入-wide $w$ period and for $\lambda / 2$-thick PML absorbers. The solid curve in Fig. 4(b) also shows the convergence performance reported in Ref. 17 obtained with the method of lines, a finite-difference modal method that is widely used for waveguide computations. The present method achieves a much better convergence rate. Similar observations were reported in Ref. 6. A thorough comparison of convergence performance between the two approaches can be found in Ref. 14, where Fourier and finite-difference modal methods for one-dimensional dielectric and metallic gratings are compared. The conclusion is clear: Expansion techniques that rely on Fourier series offer much better convergence performance than those that rely on the finite difference for diffraction problems that involve dielectric and metallic materials in the visible and the near-infrared domains.

The second example to be considered is that of a grating coupler. The grating and waveguide parameters are given in Fig. 5. We aim to compute the electromagnetic field diffracted in air by the coupler. The computation is twofold. The first step consists in computing the $S$ matrix that links the field amplitudes in the two outer media and solving for $\mathbf{u}^{(n+1)}$ and $\mathbf{d}^{(0)}$. The second step consists in computing the set of field amplitudes $\mathbf{u}^{(p)}$ and $\mathbf{d}^{(p)}$ for every layer. It deserves some care. To prevent any numerical stability problem caused by the growing exponential function, we may compute the field amplitudes in a recursive way, starting from the input region and using the following expressions:

$$
\begin{aligned}
\mathbf{d}^{(p)}= & 1 / 2 \mathbf{X}^{(p)}\left\{\left(\mathbf{W}^{(p)-1} \mathbf{W}^{(p+1)}-\mathbf{V}^{(p)-1} \mathbf{V}^{(p+1)}\right) \mathbf{u}^{(p+1)}\right. \\
& \left.+\left[\mathbf{W}^{(p)-1} \mathbf{W}^{(p+1)}+\mathbf{V}^{(p)-1} \mathbf{V}^{(p+1)}\right] \mathbf{d}^{(p+1)}\right\} \\
\mathbf{u}^{(p)}= & \mathbf{R}_{u d}^{(p-1)} d^{(p)} .
\end{aligned}
$$

These amplitudes are numerically stable. It should be mentioned that all the matrices in Eqs. (14) are already 
available from the first step. For the last step, which is related to the computation of the electromagnetic field quantities from their Fourier coefficients, it is advisable to refer to the accurate method of Ref. 19. Through appropriate use of the constitutive relations in both Fourier and real space, this method avoids possible sources of artifacts that are results of permittivity discontinuities, even in the vicinity of a wedge where field singularities exist for TM polarization.

The electromagnetic fields are then computed on a horizontal plane $\mathrm{P}$ in the air superstrate; see Fig. 5. For TE polarization, which we consider in our example, the electric field $E(z)$ in plane P can be expanded on a plane-wave basis:

$$
E(z)=\int_{-\infty}^{+\infty}\left[e_{p}\left(k_{z}\right)+e_{m}\left(k_{z}\right)\right] \exp \left(j k_{z} z\right) \mathrm{d} k_{z},
$$

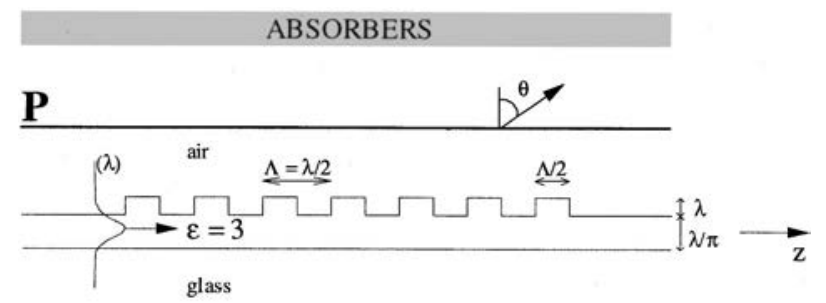

\section{ABSORBERS}

Fig. 5. Grating coupler diffraction. where $e_{m}\left(k_{z}\right)$ and $e_{p}\left(k_{z}\right)$ correspond to plane waves propagating in the negative and positive $x$ directions, respectively. Strictly speaking, $e_{m}\left(k_{z}\right)$, which corresponds to plane waves propagating toward the grating, must be null for any $k_{z}$. However, because the absorbers are not perfect (isotropic and nonmagnetic absorbers were used for this computation), we found a residual nonnull value for $e_{m}\left(k_{z}\right)$ with $\left|e_{m}\left(k_{z}\right)\right|^{2}<\left|e_{p}\left(k_{z}\right)\right|^{2} / 1000$ for any value of $k_{z}$.

The thin solid curve in Fig. 6 was obtained with the RCWA approach. It shows $\left|e_{p}(\theta)\right|^{2}$, where $\theta$ is equal to $a \sin \left(k_{z} / k_{0}\right)$, for a grating coupler length $L$ of $20 \lambda$ illuminated by the fundamental $\mathrm{TE}_{0}$ mode with normalized power. Note that the computation does not exploit the periodicity of the grating coupler in the $z$ direction. Thus, similar computations can be performed for pulsewidth-modulated gratings with outcoupling and focusing functions, for instance. The bold curve in Fig. 6 is obtained from the following formula:

$$
\left|e_{p}(\theta)\right|^{2}=A \operatorname{sinc}^{2}\left[\pi L / \lambda \cos \left(\theta_{0}\right)\left(\theta-\theta_{0}\right)\right],
$$

where $A$ is a normalizing factor set equal to the maximum value of $\left|e_{p}(\theta)\right|^{2}$ obtained with RCWA. In Eq. (16), sinc( ) is the sinc function, defined by $\sin () /()$, and $\theta_{0}$ verifies the first-order Bragg condition $n_{\text {eff }}-\lambda / \Lambda=-\sin \left(\theta_{0}\right)$, where $n_{\text {eff }}$ is the real part of the effective index of the Bloch wave of the grating waveguide $\left(n_{\text {eff }}=1.5827\right)$. The value of $n_{\text {eff }}$ is computed as explained in Ref. 6. The sinc-squared

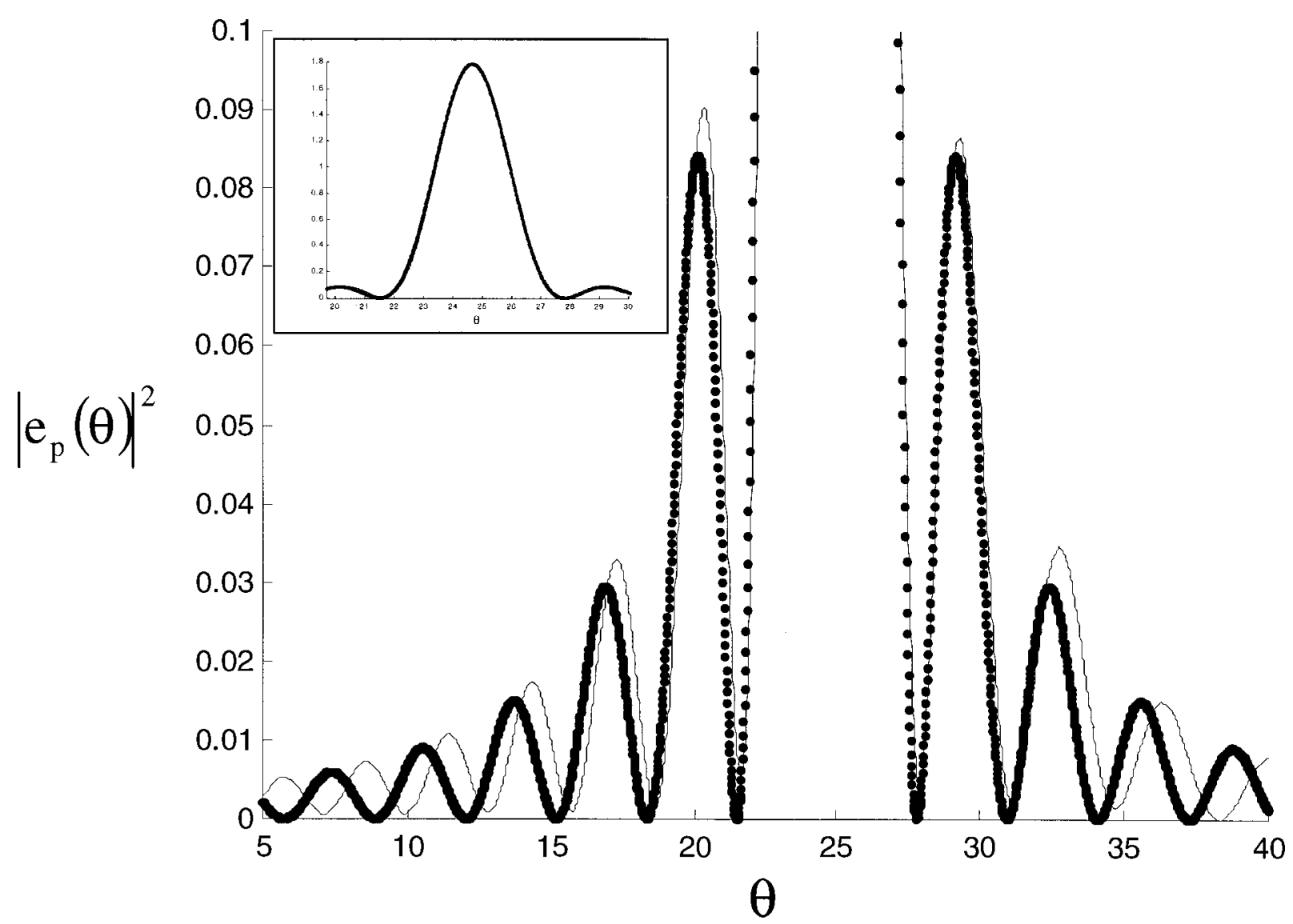

Fig. 6. Angular spectrum intensity $\left|e_{p}(\theta)\right|^{2}$ of the light diffracted in air by the grating coupler for a $20 \lambda$ grating length and for TE polarization. Thin, solid curve, present method; filled circles, the sinc-squared function of Eq. (16). Inset, enlarged view near $\theta$ $=25^{\circ}$; the filled circles and the thin curve are superimposed at this scale. 
function is an approximate expression that results from the scalar diffraction pattern of a metallic slit [slit width, $\left.L \cos \left(\theta_{0}\right)\right]$ illuminated by a plane wave. As shown in Fig. 6 , it represents a very good approximation for $\theta$ approximately equal to $\theta_{0}$.

\section{NUMERICAL RESULTS FOR THREE- DIMENSIONAL STRUCTURES}

In this section we consider more-up-to-date threedimensional problems such as the diffraction of guided waves by integrated gratings (or photonic-crystal waveguides) and waveguide airbridge microcavities. The present method is tested against both numerical and experimental data.

\section{A. Photonic-Crystal Waveguides and Integrated Gratings}

The experiment and the experimental results that we use to test the present method are described in Ref. 20, where the transmission through a finite-length photonic-crystal waveguide is measured as a function of the wavelength of the incoming guided light; see Fig. 7. We fabricated the photonic-crystal waveguide by etching small and deep holes into a planar monomode asymmetric waveguide. The waveguide is composed of a GaAs (refractive index, 3.5) 250-nm-wide core with claddings whose refractive indices are 3.4 for the cover and 3.0 for the substrate. The cover thickness is $330 \mathrm{~nm}$. The photonic-crystal waveguide consists of a triangular array of holes with a lattice parameter $\Lambda$ (distance between two adjacent holes). It is assumed to be periodic in the $x$ direction (period $\Lambda_{x}$ ) and to have a finite spatial extent into the $z$ direction. The $y$ direction (vertical index confinement) is normal to the semiconductor stack. The corrugated waveguide is assumed to be illuminated from the input unpatterned region by the fundamental $\mathrm{TE}_{0}$ or $\mathrm{TM}_{0}$ mode at an incidence angle $\theta$. We define by $f$ the air-fill factor (ratio of holes to total area). The holes are assumed to have straightsided walls (perfect cylinders) and to have a finite depth $h$. The hole depth and the fill factor are the free parameters used for fitting the calculations to the experimental data.

To solve the waveguide diffraction problem, we make the structure periodic in the $y$ direction (period $w_{y}$ is analogous to the parameter $w$ used above for twodimensional problems) and insert absorbers between the

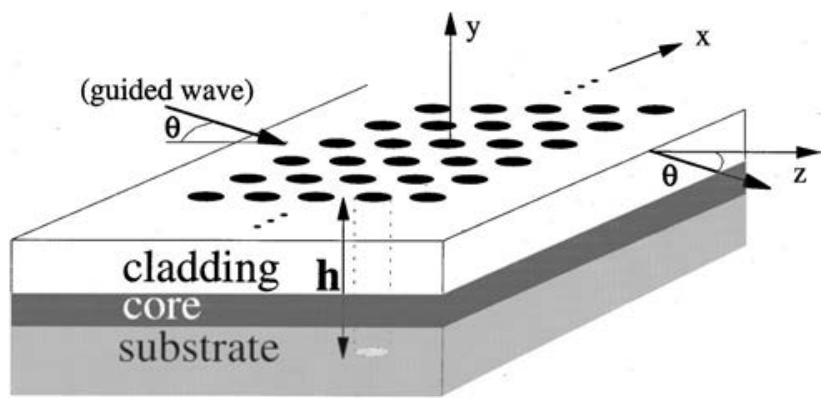

Fig. 7. Photonic-crystal waveguide geometry. The waveguide structure is periodic in the $x$ direction, has a finite arbitrary extent in the $z$ direction, and is illuminated by the fundamental mode of the unpatterned waveguide (incidence angle, $\theta$ ). waveguides. The absorber layers are parallel to the plane $y=0$. We thus obtain a biperiodic diffraction problem that is solved with the Fourier modal method of Ref. 8. Note that the electromagnetic field is looked for as a pseudoperiodic function of the $x$ coordinate (natural periodicity direction for the problem) and as a purely periodic function of the $y$ coordinate (artificial periodization direction). For instance, the magnetic field $\mathbf{H}$ may be written as

$$
\begin{aligned}
H= & \sum_{l, m}\left(U_{x l m} x+U_{y l m} y+U_{z l m} z\right) \\
& \times \exp \left[-j\left(k_{x}+l K_{x}\right) x-j m K_{y} y\right],
\end{aligned}
$$

where $k_{x}=2 \pi / \lambda n_{\text {eff }} \sin (\theta), n_{\text {eff }}$ is the effective index of the unpatterned waveguide, $K_{x}=2 \pi / \Lambda_{x}, K_{y}=2 \pi / w_{y}$, and $U_{\alpha l m}(\alpha=x, y, z)$ are the $z$-dependent normalized amplitudes of the magnetic field. We are therefore relying on a hybrid approach that mixes a natural periodicity and an artificial periodicity along the direction of index confinement. The approach is also applicable to the study of the diffraction by integrated optical gratings or arrayed-waveguide gratings that have potential applications as optical demultiplexers ${ }^{21}$ or spectrometers. ${ }^{22}$

For the numerical computations we consider that the PC waveguide is illuminated under normal incidence ( $\theta$ $=0$ ) by the fundamental $\mathrm{TE}_{0}$ mode of the waveguide propagating along the $z$ direction. Moreover, in line with the experiments, seven different lattice periods, $\Lambda$ $=180,200,220,240,260,280,300 \mathrm{~nm}$, with identical fill factors are considered. Finally, two crystal orientations are investigated such that the normally incident mode propagates along the $\Gamma M$ and $\Gamma K$ principal crystallographic axes. ${ }^{20}$ For $\Gamma M$ and $\Gamma K$, the numbers of hole rows in the $z$ direction are 8 and 15 , respectively.

Figure 8 shows a comparison of the experimental (noisy curves) and the numerical (solid curves) zero-order transmission results as a function of the normalized lattice constant $\Lambda / \lambda$. For the calculation the holes are approximated by six lamellar slices with piecewise-constant permittivities, thus replacing the actual continuous profile with a staircase profile. We use a fill factor of $f=18 \%$. This value controls the position of the band edges. Then we assume that the hole depth varies linearly from one sample to the other: $h(\Lambda)=C \Lambda+h_{0}$. This assumption (large holes are deeper than small ones) is reasonable with respect to the well-known properties of the reactiveion-etching process used for the fabrication. With $h_{0}$ $=180 \mathrm{~nm}$ and $C=1.833$, the depth varies linearly from $510 \mathrm{~nm}$ for the $180-\mathrm{nm}$-period sample to $730 \mathrm{~nm}$ for the sample with the largest period. The finite depth of the holes controls the amount of radiation $\operatorname{loss}^{23}$ (mostly in the substrate) induced by out-of-plane diffraction. Essentially, excellent agreement between the experimental and the numerical results is obtained. The band edges and the ripples on the sides of the gaps are correctly located for both $\Gamma M$ and $\Gamma K$ propagation directions. The only discrepancy between the experimental data and the model results is observed for $\Gamma K$ and for the geometry with a 0.28 normalized period. The experimental transmission is $\sim 20 \%$ smaller than the numerical prediction. Noting that the experimental results for this normalized 

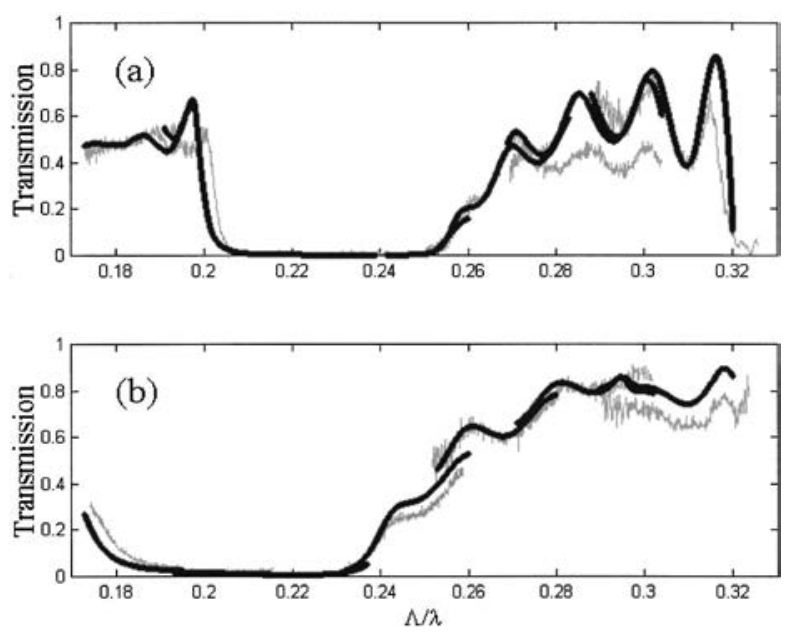

Fig. 8. Comparison of the experimental transmission measurement (noisy curves) and theoretical predictions (solid curves) for photonic-crystal diffraction as shown in Fig. 7. (a) $\Gamma K$ direction, (b) $\Gamma M$ direction.

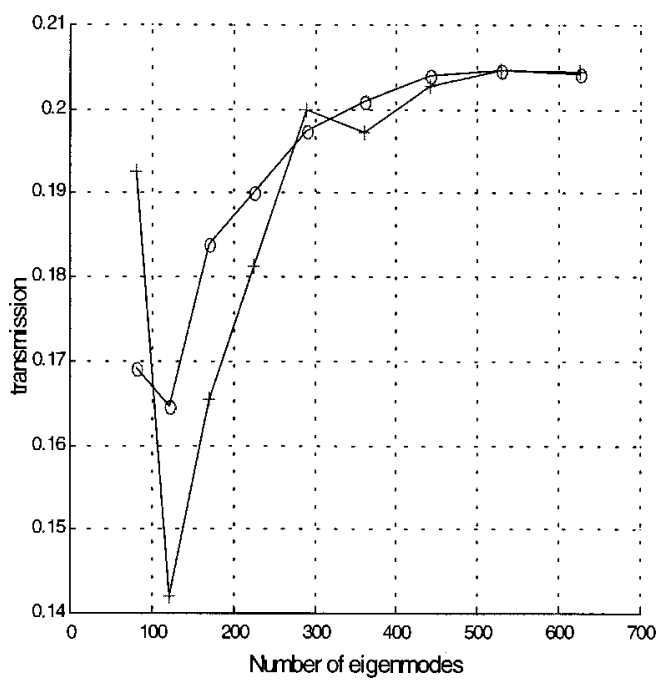

Fig. 9. Convergence performance of the present method for the photonic-crystal waveguide.

period are not consistent with those obtained for $\Lambda / \lambda$ $=0.26,0.3$, we believe that this discrepancy comes from the measurements, namely, from a local deficiency in the photoluminescence yield that is responsible for incorrect normalization of the measured data. ${ }^{20}$

Figure 9 shows the convergence performance of the present method as a function of the number of eigenmodes (or Fourier harmonics) retained for the computation. Results are given for the $\Gamma K$ case, for $\Lambda / \lambda=0.26$ $(\lambda=1 \mu \mathrm{m})$ and a hole depth of $657 \mathrm{~nm}$. The computed zero-order transmission is shown for two artificial periods, $w_{y}=2 \lambda$ and $w_{y}=3 \lambda$, that are represented by pluses and circles, respectively. For the computation, an equal number of Fourier harmonics is retained in the $x$ and $y$ directions, and we use a $0.6 \lambda$-thick absorber with a gradient-index nonmagnetic profile distribution. It is composed of two parts, A1 and A2, with identical thickness. These two parts are placed an equal distance from the center of the waveguide core. For parts A1 and A2 the refractive indices vary linearly from 1 to $1+j$ and from 3 to $3+j$, respectively. Basically, we guess from Fig. 9 that the absolute computational accuracy for the transmitted intensity is better than $\pm 1 \%$.

\section{B. Waveguide Air-Bridge Microcavities}

A diagram of the waveguide structure that we consider below is shown in Fig. 10(a). Optical waveguiding and two-dimensional confinement are generated by an airbridge structure. The air-bridge structure is etched as two sets of regularly spaced square holes that act as short Bragg reflectors. The holes are assumed to be fully etched through the waveguide. The two sets of holes create a microcavity with a resonant defect state inside the gap. The microcavity resonance peak was designed to be near the $1.5-\mu \mathrm{m}$ optical communication wavelength. The transmission responses of such small-volume cavities have already been studied experimentally for telecommunication wavelengths. ${ }^{24}$

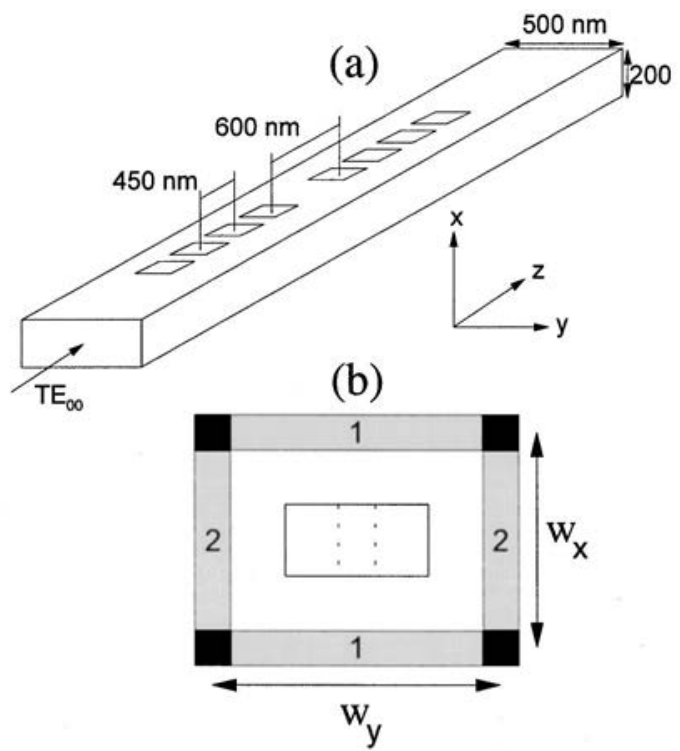

(c)

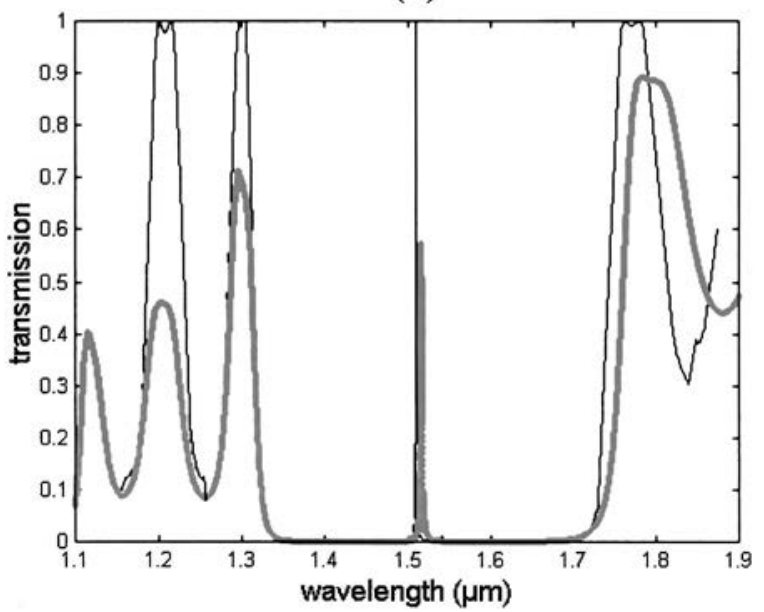

Fig. 10. Air-bridge microcavity: (a) Dimensions of the photonic bandgap air-bridge microcavity. Each square hole has a 250-nm side. (b) Computational box and absorbers. (c) Theoretical transmission of the photonic bandgap air-bridge microcavity. Thicker curve, the present method; thin curve, the finite-element modal method with perfectly conducting walls. 
For the computation we assume that the center-tocenter defect length is $600 \mathrm{~nm}$ and the center-to-center distance between two adjacent holes is $450 \mathrm{~nm}$. The relative permittivity of the air-bridge waveguide is 12.1 . The waveguide is $200 \mathrm{~nm}$ thick and $500 \mathrm{~nm}$ wide, and therefore it supports only a single TE-like mode in the wavelength ranges of interest.

To solve the electromagnetic problem we make the airbridge cavity periodic in the $x$ and $y$ directions (periods $w_{x}$ and $w_{y}$ ) and insert absorbers between the waveguides; see Fig. 10(b). The absorber layers are parallel to the $x z$ and $y z$ planes and delimit a rectangular computational box. As above, we again obtain a biperiodic diffraction problem. However, instead of through the approach of Subsection 5.A, biperiodicity is achieved through a double artificial periodicity process. Thus the electromagnetic field is looked for as a purely periodic function of the $x$ and $y$ coordinates. For instance, magnetic field $\mathbf{H}$ is written as

$$
\begin{aligned}
H= & \sum_{l, m}\left(U_{x l m} \mathbf{x}+U_{y l m} \mathbf{y}+U_{z l m} \mathbf{z}\right) \\
& \times \exp \left[-j l K_{x} x-j m K_{y} y\right],
\end{aligned}
$$

with $K_{x}=2 \pi / w_{x}$ and $K_{y}=2 \pi / w_{y}$.

For the computation, the PML absorbers are defined as in Fig. 10(b). For type 2 absorbers, $\mu_{x x}=\mu_{z z}=\varepsilon_{x x}$ $=\varepsilon_{z z}=\alpha$ and $\mu_{y y}=\varepsilon_{y y}=1 / \alpha$, where $\alpha=3(1+j)$. For type 1 absorbers, $\mu_{y y}=\mu_{z z}=\varepsilon_{y y}=\varepsilon_{z z}=\alpha$ and $\mu_{x x}=\varepsilon_{x x}=1 / \alpha$. For the four corner absorbers of Fig. 10(b), $\mu_{x x}=\mu_{y y}=\varepsilon_{x x}=\varepsilon_{y y}=1$ and $\mu_{z z}=\varepsilon_{z z}=\alpha^{2}$. It is easily verified that, for this set of parameters, the absorbers are reflectionless and the $(1+j)$ factor in the expression of $\alpha$ absorbs both evanescent and propagating waves. Every absorber in Fig. 10(b) is $0.2 \mu \mathrm{m}$ thick. The computation is performed with $w_{x}=0.8 \mu \mathrm{m}$ and $w_{y}$ $=1.1 \mu \mathrm{m}$, and for a square truncation with $M= \pm 10$ Fourier harmonics retained in each direction. It is worth mentioning that retaining 961 orders in a threedimensional Fourier modal method represents a large amount of computation. We exploited symmetry considerations similar to those reported in Ref. 25 in the code for the eigenproblem and the $S$-matrix computations to take advantage of the diffraction-problem degeneracy and to reduce memory requirements. With this approach, CPU times to compute the transmission for one frequency on a $\mathrm{PC}$ equipped with a $450-\mathrm{MHz}$ Pentium III processor are $4,15,39,86,166,294$, and $487 \mathrm{~min}$ for $\pm 5,7,9,11$, 13,15 , and 17 retained orders in each direction (see Table 2). For comparison, CPU times without exploitation of symmetry for $\pm 5,7,9$, and 11 retained orders in each direction are $5,22,68$, and $179 \mathrm{~min}$. We are not able to provide computational times for \pm 13 retained orders without exploiting any symmetry because of computer memory requirements.

The darker, thicker curve in Fig. 10(c) shows the transmission spectra of the air-bridge cavity computed with the present method. At the resonance wavelength ( $\lambda$ $\approx 1.51 \mu \mathrm{m})$, the peak transmission is $\approx 60 \%$ and the quality factor is $\approx 800$. The thinner curve in Fig. 10 (c) represents the computed transmission with a finiteelement modal method often used for microwave
Table 2. Convergence Performance for the Air-Bridge Problem

\begin{tabular}{ccc}
\hline Harmonic $(M)$ & $R$ & $T$ \\
\hline 2 & 0.5220 & 0.0075 \\
3 & 0.0056 & 0.4262 \\
4 & 0.6553 & 0.3328 \\
5 & 0.6676 & 0.2384 \\
6 & 0.8874 & 0.1517 \\
7 & 0.7358 & 0.0897 \\
8 & 0.7636 & 0.0919 \\
9 & 0.7092 & 0.0835 \\
10 & 0.7342 & 0.0872 \\
11 & 0.7298 & 0.0860 \\
12 & 0.7324 & 0.0853 \\
13 & 0.7352 & 0.0854 \\
14 & 0.7281 & 0.0843 \\
15 & 0.7319 & 0.0847 \\
\hline
\end{tabular}

engineering. ${ }^{26}$ The results are obtained with perfectly conducting walls surrounding the microcavity. As a consequence, the approach does not consider any loss by scattering, and this is why the transmission reaches unity for several frequencies. Although the two numerical methods do not deal with exactly the same diffraction problem, the comparison provides a qualitative validation of the present method. It is found that the peak transmission and the short-wavelength band edge (the one for which the losses are small) are accurately located. For the long-wavelength band edge and the ripples on the two sides of the gap, qualitative agreement is obtained.

\section{CONCLUSION}

A simple, efficient, and stable method for analyzing waveguide and grating diffraction problems is proposed. We formulated this method by extending the domain of applicability of grating theories to aperiodic structures, especially to the diffraction structures encountered in integrated optics. The method relies on an artificial periodicity of the aperiodic structures and on incorporating artificial absorbers to satisfy the ingoing wave condition. We tested the approach for several waveguide diffraction problems. These problems include twodimensional classic problems such as those associated with Bragg mirrors or grating couplers and moresophisticated three-dimensional problems such as those associated with integrated diffraction gratings (photonic crystal waveguides) and waveguide air-bridge microcavities. Both available numerical data from finitedifference and finite-element techniques and experimental data for three-dimensional problems were used for validating the approach. Excellent agreement was achieved in all cases. Rigorous coupled-wave analysis was used here to support the analysis, but we believe that the approach is applicable to other grating theories.

The results are clearly encouraging. Improvements can be expected with use of better absorbers or of coordinate transforms to rescale the direction of the artificial periodicity. From the point of view of waveguide theory, the present Fourier method belongs to the general class of 
modal spectral methods; it improves and generalizes previous studies ${ }^{6,27-29}$ that relied on the use of Fourier series in waveguide theories, and it provides solutions to problems that were not investigated previously. From the point of view of grating theory, it extends the domain of applicability of RCWA.

\section{ACKNOWLEDGMENTS}

The authors thank Pierre Chavel and Henri Benisty (Laboratoire de Physique de la Matière Condensée, Ecole Polytechnique, Palaiseau), Olivier Jacquin (Laboratoire d'Electromagnétisme, Microondes et Optoélectronique, Grenoble), and Dominique Cros, Michel Aubourg, and Olivier Piquet (Institut de Recherche en Communications Optiques et Microondes, Limoges) for fruitful discussions and for providing numerical and experimental data. This research is partly supported under the programme Microsystèmes of the Centre National de la Recherche Scientifique, France.

\section{REFERENCES AND NOTES}

1. T. Itoh, ed., Numerical Techniques for Microwave and Millimeter-Wave Passive Structures (Wiley, New York, 1989).

2. L. Li, "Recent advances and present limitations of the electromagnetic theory of diffraction gratings," in Diffractive Optics and Micro-Optics, 2000 OSA Technical Digest Series (Optical Society of America, Washington, D.C., 2000), pp. $2-4$.

3. M. G. Moharam, E. B. Grann, D. A. Pommet, and T. K. Gaylord, "Formulation for stable and efficient implementation of the rigorous coupled-wave analysis of binary gratings," $\mathrm{J}$. Opt. Soc. Am. A 12, 1068-1076 (1995).

4. F. Montiel and M. Nevière, "Differential theory of gratings: extension to deep gratings of arbitrary profile and permittivity through the $R$-matrix propagation algorithm," J. Opt. Soc. Am. A 11, 3241-3250 (1994).

5. A. Taflove, Computational Electrodynamics: The FiniteDifference Time-Domain Method (Artech House, Boston, Mass., 1995), Chap. 7.

6. Ph. Lalanne and E. Silberstein, "Fourier-modal method applied to waveguide computational problems," Opt. Lett. 25, 1092-1094 (2000).

7. Ph. Lalanne and G. M. Morris, "Highly improved convergence of the coupled-wave method for TM polarization," J. Opt. Soc. Am. A 13, 779-784 (1996).

8. L. Li, "New formulation of the Fourier modal method for crossed surface-relief gratings," J. Opt. Soc. Am. A 14, 2758-2767 (1997).

9. See, for instance, L. Li, "Formulation and comparison of two recursive matrix algorithms for modeling layered diffraction gratings," J. Opt. Soc. Am. A 13, 1024-1035 (1996).

10. G. Granet and B. Guizal, "Efficient implementation of the coupled-wave method for metallic lamellar gratings in TM polarization,” J. Opt. Soc. Am. A 13, 1019-1023 (1996).
11. L. Li, "Use of Fourier series in the analysis of discontinuous periodic structures," J. Opt. Soc. Am. A 13, 1870-1876 (1996).

12. E. Popov and M. Nevière, "Grating theory: new equations in Fourier space leading to fast converging results for TM polarization,” J. Opt. Soc. Am. A 17, 1773-1784 (2000).

13. Ph. Lalanne, "Effective properties and band structure of lamellar subwavelength crystals: plane-wave method revisited," Phys. Rev. B 58, 9801-9807 (1998).

14. Ph. Lalanne and J. P. Hugonin, "Numerical performance of finite-difference modal methods for the electromagnetic analysis of one-dimensional lamellar gratings," J. Opt. Soc. Am. A 17, 1033-1042 (2000).

15. J. P. Bérenger, "A perfectly matched layer for the absorption of electromagnetic waves," J. Comput. Phys. 114, 185-200 (1994).

16. See, for instance, E. A. Marengo, C. M. Rappaport, and E. L. Miller, "Optimum PML ABC conductivity profile in FDTD," IEEE Trans. Magn. 35, 1506-1509 (1999).

17. S. F. Helfert and R. Pregla, "Efficient analysis of periodic structures," J. Lightwave Technol. 16, 1694-1702 (1998).

18. R. Pregla and W. Pasher, "The method of lines," in Numerical Techniques for Microwave and Millimeter Wave Passive Structure, T. Itoh, ed. (Wiley, New York, 1989), pp. 381-446.

19. Ph. Lalanne and M. P. Jurek, "Computation of the nearfield pattern with the coupled-wave method for TM polarization," J. Mod. Opt. 45, 1357-1374 (1998).

20. D. Labilloy, H. Benisty, C. Weisbuch, T. F. Krauss, R. M. De La Rue, V. Bardinal, R. Houdré, U. Oesterle, D. Cassagne, and C. Jouanin, "Quantitative measurement of transmission, reflection and diffraction of two-dimensional photonic bandgap structures at near-infrared wavelengths," Phys. Rev. Lett. 79, 4147-4150 (1997).

21. M. S. D. Smith and K. A. McGreer, "Diffraction gratings utilizing total internal reflection facets in Littrow configuration,” IEEE Photon. Technol. Lett. 11, 84-86 (1999).

22. J. B. D. Soole, A. Scherer, H. P. LeBlanc, N. C. Andreadakis, R. Bhat, and M. A. Kosa, "Monolithic InP/InGaAsP/InP grating spectrometer for the 1.48-1.56 $\mu \mathrm{m}$ wavelength range," Appl. Phys. Lett. 58, 1949-1951 (1991).

23. Ph. Lalanne and H. Benisty, "Out-of-plane losses of twodimensional photonic crystal waveguides: electromagnetic analysis," J. Appl. Phys. 89, 1512-1514 (2001).

24. D. J. Ripin, K. Y. Lim, G. S. Petrich, P. R. Villeneuve, S. Fan, E. R. Thoen, J. D. Joannopoulos, E. P. Ippen, and L. A. Kolodziejski, "Photonic band gap airbridge microcavity resonances in GaAs $/ \mathrm{Al}_{\mathrm{x}} \mathrm{O}_{\mathrm{y}}$ waveguides," J. Appl. Phys. 87, 1578-1580 (2000)

25. Ph. Lalanne and D. Lalanne, "On the effective medium theory of subwavelength periodic structures," J. Mod. Opt. 43, 2063-2086 (1996).

26. M. Aubourg, S. Verdeyme, and P. Guillon, "Finite element software for microwave engineering," in Microwave Passive Devices, T. Itoh, G. Pelosi, and P. P. Silvester, eds. (Wiley, New York, 1996), Chap. 1.

27. C. H. Henry and B. H. Verbeek, "Solution of the scalar wave equation for arbitrarily shaped dielectric waveguides by two-dimensional Fourier analysis," J. Lightwave Technol. 7, 308-313 (1989).

28. J. Rodriguez, R. D. Crespo, S. Fernandez, J. Pandavenes, J. Olivares, S. Carrasco, I. Ibanez, and J. M. Virgos, "Radiation losses on discontinuities in integrated optical waveguides," Opt. Eng. 38, 1896-1906 (1999).

29. P. Vahihama and J. Turunen, Diffractive Optics and MicroOptics, Vol. 10 of 1998 OSA Technical Digest Series (Optical Society of America, Washington, D. C., 1998), p. 69. 